NOTA DE PESQUISA

\title{
Haemaphysalis juxtakochi COOLEY, 1946 (ACARI: IXODIDAE) PARASITANDO Mazama nana (HENSEL, 1872) (ARTIODACTYLA: CERVIDAE) NO ESTADO DO RIO GRANDE DO SUL*
}

\author{
JOÃO RICARDO MARTINS'; EDSON LUÍS SALOMÃO ${ }^{2}$; ROVAINA L. DOYLE ${ }^{1}$; VALÉRIA ONOFRIO ${ }^{3}$, \\ DARCI M. BARROS-BATTESTI ${ }^{3}$, ALBERTO A. GUGLIELMONE ${ }^{4}$
}

\begin{abstract}
MARTINS, J.R.; SALOMÃO, E.L.; DOYLE, R.L.; ONOFRIO, V.C.; BARROS-BATTESTI, D.M.; GUGLIELMONE, A.A. [Haemaphysalis juxtakochi Cooley, 1946 (Acari: Ixodidae) parasitizing Mazama nana (Hensel, 1872) (Artiodactyla: Cervidae) in the State of Rio Grande do Sul.] Haemaphysalis juxtakochi Cooley, 1946 (Acari: Ixodidae) parasitando Mazama nana (Hensel, 1872) (Artiodactyla: Cervidae) no Estado do Rio Grande do Sul. Revista Brasileira de Parasitologia Veterinária, v. 16, n. 3, p. 171-173, 2007. Instituto de Pesquisas Veterinárias Desidério Finamor, Estrada do Conde, 6000. Eldorado do Sul, RS, Brasil. 92990-000. E-mail: joaorsm@terra.com.br

The record of Haemaphysalis juxtakochi Cooley, an ixodid tick, on the brocket deer, Mazama nana (Hensel), represents a new report after 34 years of its last report in the State of Rio Grande do Sul. The tick specimens (three males and one female) were found in Cachoeira do Sul, RS (3002'21"S, 52 $53^{\circ} 38^{\prime \prime} \mathrm{W}$ ). This place is elevated $72 \mathrm{~m}$ sea above, with an annual average temperature of $18.8^{\circ} \mathrm{C}$, and annual average rainfall of $1.438 \mathrm{~mm}$. This is the first record of this tick species in a Cervidae and it is the second occurrence report for the State of Rio Grande do Sul.
\end{abstract}

KEY WORDS: Haemaphysalis juxtakochi, Ixodidae, Mazama nana, Cervidae, Rio Grande do Sul.

\section{RESUMO}

O encontro de Haemaphysalis juxtakochi Cooley, um carrapato ixodídeo, no veado bororó-do-sul, Mazama nana (Hensel), representa novo relato de ocorrência, após 34 anos de seu registro no Estado do Rio Grande do Sul. Os exemplares de carrapatos (3 machos e 1 fêmea) foram encontrados em Cachoeira do Sul, RS (3002’21"S, 5253’38"W). O local está a $72 \mathrm{~m}$ acima do nível do mar, com uma média anual de temperatura de $18,8^{\circ} \mathrm{C}$, e uma precipitação média anual de 1.438 mm. Este é o primeiro registro de $H$. juxtakochi nesta espécie

\footnotetext{
${ }^{*}$ Apoio FAPESP (processo No. 99/05446-8) e CNPq (processo No. 478950/2004-7).

${ }^{1}$ Instituto de Pesquisas Veterinárias Desidério Finamor. Estrada do Conde, 6000, Eldorado do Sul, RS 92990-000. E-mail: joaorsm@terra.com.br

${ }^{2}$ Clínica Veterinária Dr. Edson Salomão, Cachoeira do Sul, RS.

${ }^{3}$ Instituto Butantan. Av. Vital Brasil, 1500, São Paulo, SP 05503-900.

${ }^{4}$ Instituto Nacional de Tecnología Agropecuaria (INTA), Estación Experimental de Rafaela, Rafaela, CC 22, Santa Fé, Argentina.
}

de cervídeo e um segundo relato de ocorrência para o Estado do Rio Grande do Sul.

PALAVRAS-CHAVE: Haemaphysalis juxtakochi, Ixodidae, Mazama nana, Cervidae, Rio Grande do Sul.

O gênero Haemaphysalis é o segundo maior gênero de Ixodidae em espécies no mundo. Dentre elas, somente três espécies ocorrem na região Neotropical: H. cinnabarina Koch, 1844, que é uma espécie conhecida apenas pelos espécimes tipo, originalmente descrita do Estado do Pará de hospedeiro ignorado; H. leporipalustris (Packard, 1869), comumente encontrada em coelhos silvestres e H. juxtakochi Cooley, 1946 que parasita preferencialmente mamíferos da família Cervidae (ONOFRIO et al., 2006).

O prévio relato conhecido de $H$. juxtakochi para o Rio Grande do Sul, corresponde a uma ocorrência registrada por Freire (1972) no veado-pardo ou veado-virá, Mazama sp., capturado no município de Arroio Grande (32 $\left.{ }^{\circ} 14^{\prime} \mathrm{S}, 5^{\circ} 05^{\prime} \mathrm{W}\right)$. 




Figura1. Haemaphysalis juxtakochi Cooley, macho. Gnatossoma ventral, em destaque a expansão lateral do artículo II (fêmur), o prolongamento ventral do artículo III (genu) dos palpos e o hipostômio com dentição 4/4, $=60 \mu \mathrm{m}$.

Figura 2. Coxas I-IV, em destaque a base retangular do capítulo e os espinhos dos trocânteres, $=300 \mu \mathrm{m}$.

Curiosamente esse autor também mencionou a presença de um exemplar de $H$. leporispalustris em M. americana (Erxleben) capturado em Três Passos. No entanto, esse relato é sugestivo de um eventual equívoco já que esta espécie de carrapato, no estágio adulto, parasita preferencialmente lagomorfos, embora os estágios imaturos sejam freqüentes em aves (KINSEY et al., 2000; BELDOMENICO et al., 2003). Evans et al. (2000) mencionaram ambos os registros de Freire (1972), mas enfatizaram a necessidade de novos encontros para confirmar a atual existência dessas espécies no Estado do Rio Grande do Sul.

Somente recentemente, durante a vistoria da pelagem de Mazama nana (Hensel), popularmente conhecida como veado-bororó-do-sul, foram retirados 3 machos e uma fêmea de H. juxtakochi confirmando assim a ocorrência desta espécie para o Rio Grande do Sul. O animal foi capturado no municí-

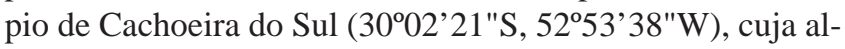
titude média é de $72 \mathrm{~m}$ acima do nível do mar, com uma média anual de temperatura de $18.8^{\circ} \mathrm{C}$, e uma precipitação média anual de $1.438 \mathrm{~mm}$. Os carrapatos foram identificados através da chave dicotômica de Aragão (1936) e depositados na coleção de carrapatos do Instituto de Pesquisas Veterinárias Desidério Finamor, Eldorado do Sul.

Dentre as características peculiares na morfologia desta espécie de carrapato, destacam-se a existência de um longo prolongamento retrógrado ventral no $3^{\circ}$ artículo (genu) dos palpos, e a presença de 4 ou 5 fileiras de dentes de cada lado da linha média do hipostômio (Figura 1). Outros aspectos morfológicos desta espécie comuns a outras do gênero são: expansão lateral do $2^{\circ}$ artículo (fêmur) dos palpos, base retangular do capítulo e a presença de espinhos nos trocânteres das coxas I-IV (Figuras 1 e 2).

Embora tenha sido registrada em roedores, anta, quati e porcos selvagens do Panamá e Venezuela (FAIRCHILD et al., 1966; JONES et al., 1972), H. juxtakochi realiza seu ciclo biológico utilizando três hospedeiros, preferencialmente mamíferos da família Cervidae. Na Argentina, há registros de parasitismo em cão doméstico, bovino, suíno e em aves da família Corvidae (BOERO, 1957; GUGLIELMONE et al., 1992; BELDOMENICO et al., 2003). No Brasil, sua ocorrência abrange os Estados de Pernambuco, Rondônia, Mato Grosso, Minas Gerais, Rio de Janeiro, São Paulo, Paraná e Rio Grande do Sul (ARAGÃO, 1936; KOHLS, 1960; FREIRE, 1972; BARROS; BAGGIO, 1992; SERRA-FREIRE et al., 1996; ARZUA et al., 2005; LABRUNA et al., 2005). Além de Cervidae e outros grupos de mamíferos, $H$. juxtakochi foi também assinalada em ave da espécie Pyrrhocoma ruficeps (Strickland) (Passeriformes: Emberizidae) por Arzua et al. (2005), e acidentalmente, pode parasitar humano (GUGLIELMONE et al., 2006).

Do ponto de vista epidemiológico, a espécie $H$. juxtakochi pode potencialmente participar de ciclos enzoóticos na natureza. Labruna et al. (2005) reportaram o isolamento da bactéria da Rickettsia rhipicephali em exemplares de H. juxtakochi provenientes de Rondônia. Segundo Labruna e Machado (2006), apesar desta bactéria não causar doença em humanos e animais, a história mostra que nenhuma espécie de Rickettsia deve ser considerada não patogênica, até que se mostre o contrário.

Este é o primeiro registro de $H$. juxtakochi em $M$. nana e um segundo relato de ocorrência para o Rio Grande do Sul. Dessa forma, a presente nota contribui para o conhecimento da distribuição geográfica de $H$. juxtakochi e confirma sua presença neste Estado, após um período de 3 décadas sem notificação.

\section{REFERÊNCIAS BIBLIOGRÁFICAS}

ARAGÃO, H.B. Ixodidas brasileiros e de alguns paizes limitrophes. Memórias do Instituto Oswaldo Cruz, v. 31, n.4, p.759-843, 1936. 
ARZUA, M.; ONOFRIO, V.C.; BARROS-BATTESTI, D.M. Catalogue of the tick collection (Acari, Ixodida) of the Museu de Historia Natural Capão da Imbuia, Curitiba, Paraná, Brazil. Revista Brasileira de Zoologia, v. 22, n. 3, p. 623-632, 2005.

BARROS, D.M.; BAGGIO, D. Ectoparasites Ixodida Leach, 1817 on wild mammals in the State of Paraná, Brazil. Memórias do Instituto Oswaldo Cruz, v. 87, n. 2, p. 291-296, 1992.

BELDOMENICO, P.M.; BALDI, C.J.; ANTONIAZZI, L.R.; ORDUNA, G.M.; MASTROPAOLO, M.; MACEDO, A.C.; RUIZ, M.F.; ORCELLET, V.M.; PERALTA, J.L.; VENZAL, J.M.; MANGOLD, A.J.; GUGLIELMONE, A.A. Ixodid ticks (Acari: Ixodidae) present at Parque Nacional El Rey, Argentina. Neotropical Entomology, v. 32, n. 2, p. 273-277, 2003.

BOERO, J.J. Las garrapatas de la República Argentina (Acarina:Ixodidae). Buenos Aires: Universidad de Buenos Aires, Depto. Editorial, 1957. 113 p.

EVANS, D.E.; MARTINS, J.R.; GUGLIELMONE, A.A. A review of the ticks (Acari, Ixodida) of Brazil, their hosts and geographic distribution - 1. The State of Rio Grande do Sul, Southern Brazil. Memórias do Insituto Oswaldo Cruz, v. 95, n. 4, p. 453-470, 2000.

FAIRCHILD, G.B.; KOHLS, G.M.; TIPTON, V.J. The ticks of Panamá (Acarina: Ixodoidea). In: WENZEL, R.L.; TIPTON, V.J. (Eds). Ectoparasites of Panama. Chicago: Field Museum of Natural History, 1966. p.167-219.

FREIRE, J.J. Revisão das espécies da família ixodidae. Revista de Medicina Veterinária, v. 8, n. 1, p. 1-16, 1972.

GUGLIELMONE, A.A.; MANGOLD, A.J.; AUFRANC, C.R. Haemaphysalis juxtakochi, Ixodes pararicinus (Ixodidae) and Otobius megnini (Argasidae) in relation to the phytogeography of Argentina. Annales de Parasitologie Humaine et Comparee, v. 67, n. 3, p. 91-93, 1992.

GUGLIELMONE, A.A.; BEATI, L.; BARROS-BATTESTI, D.M.; LABRUNA., M.B.; NAVA, S.; VENZAL, J.M.; MANGOLD, A.J.; SZABÓ, M.P.J.; MARTINS, J.R.;
GONZÁLES-ACUÑA, D.; ESTRADA-PEÑA, A.A. Ticks (Ixodidae) on humans in South America. Experimental and Applied Acarology v. 40, n. 2, p.83-100, 2006.

JONES, E.K.; CLIFFORD, C.M.; KEIRANS, J.E.; KOHLS, G.M. The ticks of Venezuela (Acarina: Ixodoidea) with a key to the species of Amblyomma in the western hemisphere. 4 ed. Provo: Brigham Young University Science Bulletin, 1972. p. 38.

KINSEY, A.A.; DURDEN, L.A.; OLIVER, J.H. Tick infestations of birds in coastal Georgia and Alabama. Journal of Parasitology, v. 86, n. 2, p. 251-254, 2000.

KOHLS, G.M. Records and new synonymy of new world Haemaphysalis ticks, with descriptions of the nymph and larva of $H$. juxtakochi Cooley. Journal of Parasitology, v. 46, n. 3, p. 355-361, 1960.

LABRUNA, M.B.; CAMARGO, L.M.A.; CAMARGO, E.P.; WALKER, D.H. Detection of a spotted fever group Rickettsia in the tick Haemaphysalis juxtakochi in Rondonia, Brazil. Veterinary Parasitology, v. 127, n. 2, p. 169-174, 2005.

LABRUNA, M.B.; MACHADO, R.Z. Agentes transmitidos por carrapatos na região neotropical. In: BARROSBATTESTI, D.M. ARZUA, M.; BECHARA G.H. (Ed.). Carrapatos de Importância Médico-Veterinária da Região Neotropical: um guia ilustrado para a identificação de espécies. São Paulo: Vox/ICTTD-3/Butantan, 2006. p.155164.

ONOFRIO, V.C.; VENZAL, J.M.; PINTER, A.; SZABÓ, M.P.J. Família Ixodidae: características gerais, comentários e chave para gêneros. In: BARROS-BATTESTI, D.M. ARZUA, M.; BECHARA G.H. (Ed.). Carrapatos de Importância Médico-Veterinária da Região Neotropical: um guia ilustrado para a identificação de espécies. São Paulo: Vox/ICTTD-3/Butantan, 2006. p. 29-39.

SERRA-FREIRE, N.M.; AMORIM, M.; GAZETA, G.S.; GUERIM, L.; DESIDÉRIO, M.H.G. Ixodidae of deer in Brazil. Revista Brasileira de Ciência Veterinária, v. 3, n. 1, p. 51-54, 1996.

Recebido em 07 de fevereiro de 2007.

Aceito para publicação em 03 de agosto de 2007. 